1 A novel approach to analyzing all trials in chronometric mental rotation

2 and description of a flexible extended library of stimuli

3 Leonardo Jost and Petra Jansen

4 University of Regensburg

5

6 Author Note

7 Leonardo Jost, Faculty of Human Sciences, University of Regensburg;

8 Petra Jansen, Faculty of Human Sciences, University of Regensburg.

9 Correspondence concerning this article should be addressed to Leonardo Jost, Faculty of

10 Human Sciences, University of Regensburg, Universitätsstraße 31, 93053 Regensburg,

11 Germany.

12 Email: Leonardo.jost@ur.de ORC ID: 0000-0003-3508-4315

13

14 This is an Accepted Manuscript of an article published by Taylor \& Francis in Spatial

15 Cognition \& Computation: An Interdisciplinary Journal on 03 May 2020, available online:

16 https://www.tandfonline.com/doi/full/10.1080/13875868.2020.1754833 


\section{A novel approach to analyzing all trials in chronometric mental rotation 18 and description of a flexible extended library of stimuli}

Mirrored stimuli in chronometric mental rotation tests cannot be rotated into congruence and are typically discarded from analysis. We present a novel design using three figures such that congruence is achieved for all trials. For design construction we consider the geometrical properties of rotation and mirroring.

We have experimentally tested the approach with forty-one German sport students. comparable to the classical two-figure design. Based on our findings we suggest our new layout for future chronometric mental rotation. This will improve analysis of accuracies while increasing power. stimuli, which we make available on GitHub at https://github.com/LeonardoJost/MRlibrary. 
Introduction

Spatial abilities are important in everyday activities and they are related to various abilities like mathematical ability (Xie, Zhang, Chen, \& Xin, 2019) or problem solving (Geary, Saults, Liu, \& Hoard, 2000). An important spatial ability, especially an intrinsicdynamic ability according to the classification of Uttal et al. (2013), is mental rotation, the skill to imagine rotated objects in mind. An increased mental rotation performance is related to the attendance of a high school with many spatial school subjects such as chemistry, design, or biology (Moè, 2016) or a STEM degree (Hausmann, 2014). Due to the significance of the mental rotation performance it seems to be necessary to design well-defined mental rotation test to investigate this specific spatial performance. Because the ability to mentally rotate items is not only of theoretical scientific interest but relates for example to STEM education (Moè, Jansen, \& Pietsch, 2018), it is the main goal of this study to describe a new experimental mental rotation design, with which all trials can be analyzed.

To investigate the mental rotation ability, psychometric (paper-pencil) and chronometric (computer based) tasks exist. Until now, in classical chronometric mental rotation tests (Shepard \& Metzler, 1971), participants are presented two figures and they have to mentally rotate one figure to decide if the figures are the "same", which means they can be rotated into congruence, or "different", which means mirror-reversed. This decision of nonmirrored or mirror-reversed object requires an object-based transformation (Voyer, Jansen, \& Kaltner, 2017). In this kind of transformation the observer's position remains fixed and the object is moved mentally in relation to the surrounding environment. In the other class of mental transformations, egocentric transformations, figures, which typically are human

54 bodies or body parts, are processed by a transformation of one's own perspective (Zacks \& Tversky, 2005). However, mental rotation can be dissociated from the ability to reorient the imagined self, which is mostly measured by perspective-taking tests (Kozhevnikov \& 
Hegarty, 2001). A highly discussed issue in mental rotation research is the existence of gender differences. However, there is an ongoing debate as to whether and how gender influences chronometric mental rotation tests (Jansen-Osmann \& Heil, 2007). Next to influences on performance of stimulus properties such as dimensionality (Jolicœur, Regehr, Smith, \& Smith, 1985; Shepard \& Metzler, 1988), the role of distractors (Boone \& Hegarty, 2017) and interpreting results regarding the usage of different strategies (Khooshabeh \& Hegarty, 2010) to date, there is one main methodological concern in the use of chronometric mental rotation tests: Because mirrored figures cannot be brought into congruence those items have been deleted mostly from analysis so far (Jolicœur et al., 1985, Shepard \& Metzler, 1971). This incongruence is rarely further analyzed but is reflected in reaction time differences of almost one second in the analysis of Shepard and Metzler (1971) and around 500ms for cube figures in Voyer and Jansen (2016). As mirrored figures typically contribute about half of the tasks, disregarding them means losing a noticeable amount of power or testing time in experiments (Brysbaert \& Stevens, 2018). Moreover, analyzing accuracy only on non-mirrored stimuli disregards both false alarms and correct rejects, which might show a different pattern than hits and misses. Analyzing sensitivity instead of accuracy incorporates false alarms (Stanislaw, \& Todorov, 1999). However, as false alarms occur only on mirrored stimuli, they cannot be matched to an angle of rotation and only global analyses of sensitivity are appropriate.

To overcome the shortcoming of the former chronometrical mental rotation tests we present a novel approach, in which participants are shown three figures instead of two. A similar approach has already been used by Wohlschläger and Wohlschläger (1998) and by two subsequent studies from Wohlschläger (2001) and Krüger and Krist (2009) while investigating the common processing of manual and mental rotation. However, because they 
between mirrored and non-mirrored items. They found that reaction time averaged across their mirrored and non-mirrored stimuli demonstrated an increase with increasing angular disparity. In the design presented here we will call two figures the base figures, which are mirrored to each other, and the third the stimulus figure, which is a rotated version of one of the base figures. Instead of a same/different decision, participants are tasked to find out if the stimulus can be rotated into congruence with one or the other base figure. As the base figures are mirrored to each other and not mirror-symmetrical, the stimulus is congruent to one and only one base figure. Thus, the rotation into congruence is independent of stimulus

90 orientation.

\section{Goal of the study}

92 As mentioned above, the main goal of this study is to evaluate the new proposed mental rotation paradigm. While in theory the task at hand can be solved by ignoring one base figure and proceeding as in the case of two figures, we believe that presentation of a unique $0^{\circ}$ condition for all stimuli will show the same behavior for both same and different stimuli (or matching the left or the right base figure in this study). Moreover, we believe that for all stimuli the same mental transformations as in the two-figure case are performed and we expect to find the well-known mental rotation effects: An increase of reaction time and a

99 decrease of accuracy with increased angles of rotation (Shepard \& Metzler, 1971).

100 Furthermore, we predict that participants improve over time with larger improvements for

101 larger degrees. By design, we expect these effects not to differ by the side of the correct answer, although we presume a possible effect due to the left/right arrangement in accordance

103 with the reading direction of our participants. Because of the geometric considerations of the

104 rotation axes presented in the method section, we hypothesize to find no differences of the

105 aforementioned effects between rotation axes. As a secondary hypothesis, we expected to 
106 find better performances for participants with previous experience in chronometric mental

107 rotation experiments. At least, short-term benefits for mental rotation training on mental

108 rotation tasks have been shown so far (Meneghetti, Cardillo, Marmarella, Caviola, \& Borella,

109 2017). Due to the debate on gender differences in chronometric mental rotation tests (e.g.

110 Jansen-Osmann \& Heil, 2007) gender was not regarded as an additional factor.

111 In addition to the empirical evaluation of the test, we have created an extended,

112 flexible library of stimulus material and describe its properties and utilization.

\section{Method}

\section{Participants}

115 A total of 41 German students (32 females, 9 males) participated in this study due to study

116 credit. On average, participants were 21.3 years old $(\mathrm{SD}=1.8)$. In our sample, we included

117 participants with self-reported previous experience in mental rotation experiments employing

118 the two-figure design (24 with experience, 17 without). For appropriate power, Brysbaert and

119 Stevens (2018) recommend at least 1600 observations per condition independent of effect

120 sizes of other work. As we expected to exceed an average of 40 stimuli per participant per

121 condition in our design, this should yield high power for the main hypotheses. Supporting this

122 we found 14447 usable measurements in the smallest dataset equivalent to 1805 observations

123 per condition for the triple interactions of interest. For the secondary hypotheses, Meneghetti

124 et al (2017) report large effect sizes of $d>1$ for transfer within chronometric mental rotation.

125 G*power (Faul, Erdfelder, Lang, \& Buchner, 2007) shows a power of .80 for a between 126 subjects t-test at an effect size of $\mathrm{d}=0.8$.

\section{Stimulus library}

128 Due to the work of Peters and Battista (2008) a stimulus library of cube figures is already 
129 available. In an attempt to expand the applications and research possibilities, we have written

130 an extended, more flexible generation process in R (R Core Team, 2018). To keep

131 comparisons with older work valid, we have used the same cube structure for the base figures

132 as Peters and Battista (2008), although we do not exactly reproduce the rotated versions. In

133 the supplementary material, we describe the reasoned small changes we have made as well as

134 possible extensions and applications. Both pregenerated cube figures and the code for

135 generation of individual figures are available at https://github.com/LeonardoJost/MRlibrary.

136 Upon usage, we advise to state all values of the parameters given in table 1.

\section{Rationale for figure layout}

138 Different layouts are possible to achieve the proposed mental rotation task with three figures

139 with some possibilities presented in figure 1 and many more possible layouts not depicted.

140 We have decided to present the three figures arranged as in figure 1a and 4 for three

141 following main reasons: First, the usage of screen space allows larger images while avoiding

142 overlapping. Second, the layout provides congruence between hand position, response key

143 layout, and figure placement (left/right). Third, the optical distances between stimuli and base

144 figures are small and comparable, while also keeping discrimination between base and

145 stimulus figures clear for participants.

\section{Geometric considerations of axes and mirroring planes in three dimensions}

148 The necessity for the development of the new test presented here is due to the incongruence

149 of mirrored stimuli. In this section, we will review this incongruence and describe

150 geometrical properties of mirroring planes and rotation axes, which we have used as the basis

151 for our design. For the most part, we only consider the natural planes and axes for mirroring

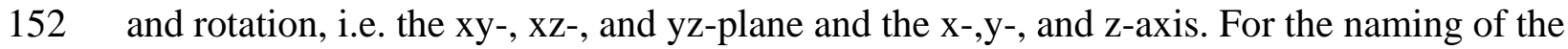


axes, we have used the convention that the vertical axis is the z-axis with larger values for

154 higher points, the horizontal axis is the $\mathrm{x}$-axis with larger values for points further to the right

155 and the depth axis is the y-axis with larger values for points further back. If these axes pass

156 through the center of an object with the front pointing towards the observer, the resulting

157 spins are known as pitch for the rotation around the $\mathrm{x}$-axis, roll for the rotation around the $\mathrm{y}$ -

158 axis, and yaw for the rotation around the z-axis. It also has to be noted, that mirrored stimuli

159 are images of the mirrored figure. As base rotation angles are employed in the $0^{\circ}$-position,

160 these are not actual mirror images. This is demonstrated in figure 2, where images of

161 mirrored figures are shown. While the three-dimensional figures are mirrored to each other,

162 the two-dimensional images of them are not.

163 Incongruence of mirrored stimuli

164 While a mirrored figure is clearly unique in shape, depending on the orientation of the

165 mirroring plane, the mirror image appears at different rotated positions. In figure 2 , the

166 images resulting from a mirroring across the xy-plane and the yz-plane differ by a rotation

167 around the y-axis by $180^{\circ}$. More generally, two mirror figures deviate by twice the angle at

168 which the two mirroring planes are rotated to each other. As a result, there exists no unique

$1690^{\circ}$-position and thus no angular disparity for mirrored stimuli. This problem also occurs in

170 two dimensions, as rotation of mirroring lines also produces rotated stimuli. A case, in which

171 this incongruence might be lessened are for example pictures of human or animal figures,

172 where a clear upright position exists, or mental rotation tasks with egocentric transformations.

173 Rotations around the z-axis, which maintain the upright position, might still cause problems,

174 although one might argue in favor of a unique front facing position, which would also solve

175 this incongruence. 
178 As we choose to present two mirrored base figures and by the incongruence no unique way of 179 choosing the mirrored figure exists, the question of choosing a base orientation arises. We 180 propose to use the mirroring plane that optically lies between the two base figures, such that 181 they can more easily be recognized as mirrored shapes of each other. As a result, we use the 182 yz-plane for the mirroring in our layout, while e.g. in the layout of figure 1c we would use the 183 xy-plane. As we cannot depict a layout in depth on a two dimensional screen, we ignore mirroring across the xz-plane in this step. Moreover, mirroring switches the image along one axis while rotation keeps one axis fixed. Mirroring across one plane switches the values along the axis that is perpendicular to

187 that plane, while keeping values constant along the axes that span the plane. Rotation on the 188 other hand keeps the coordinates along the rotation axis constant, while the other two change. 189 As can be seen in figure 2, mirroring across the yz-plane switches the coordinates along the $\mathrm{x}$-axis, while keeping the others the same, i.e. left and right are reversed. The rotation can be

191 seen in figure 3, where, as the figure is rotated around the x-axis, the left and right parts do 192 not change, but front/back and top/bottom are moved by the rotation. As a result, if a

193 mirroring across the yz-plane and a rotation around the $\mathrm{x}$-axis were used and the rotation axis were known, no rotation strategy would be necessary to solve the task, as a simple left/right comparison would be sufficient. Thus, it seems sensible to choose the rotation axes from the axes that span the mirroring plane. In our case of the yz-plane these are the $y$ - and the z-axis,

197 as we restrict ourselves to the natural axes.

198 Another problem with a combination of mirroring and rotation is demonstrated in

199 figure 4 . A rotation by $180^{\circ}$ around the $y$-axis of the left base figure can easily identified as a 200 mirroring of the right base figure across the xy-plane and similarly a rotation around the z201 axis as a mirroring across the xz-plane. Because participants will be informed that only one 
202 side can be correct and that the two base figures are mirrored to each other, the task can be

203 solved by exclusion. While this strategy is demonstrated in the case of a rotation by $180^{\circ}$, it is

204 theoretically applicable to all rotation angles, but reaches the natural mirroring planes only at

$2050^{\circ}$ and $180^{\circ}$ (and for a rotation of $0^{\circ}$ a much simpler solution is available as two figures are

206 identical). As generally larger reaction times are found in the case of mirrored stimuli in

207 classical mental rotation (Shepard \& Metzler, 1971; Voyer \& Jansen, 2016), where the same

208 strategy is applicable, this strategy seems too complex to have a major impact on results.

209 On a further note, the three axes differ in dimension, as rotation around the y-axis is

210 only a rotation in the picture plane, while the $\mathrm{x}$ - and $\mathrm{z}$-axis produce rotations in depth, but

211 typically no systematic differences between in-depth rotation and picture-plane rotation are

212 found (Shepard \& Metzler, 1971).

Please insert figure 4 about here

214 Material

Mental Rotation

216 Stimulus presentation and response handling were controlled with Presentation ${ }^{\circledR}$ software

217 (Version 20.1 Build 12.04.17, Neurobehavioral Systems, Inc., Berkeley, CA,

218 www.neurobs.com) on a Dell Latitude E7240 Laptop (12.5” screen, 1366x768, 60Hz).

219 Participants were seated in front of a desk with the laptop on it and were free to adjust both

220 the laptop and their seating position.

The stimuli were generated from the stimulus library with the parameters given in table 1. Stimulus images were then resized to 400px times 400px and presented according to the layout in figure 1a and 4 until a response was given. The vertical shift of the images was

224 set to 150 pixels (above the center for the base figures and below the center for the stimulus

225 figure) and the horizontal shift to 300 pixels (left and right of the center for the base figures). 
right, $\boldsymbol{X}$ - wrong) shown at the center of the screen at font size 40 . In the main session, participants received no feedback and a fixation cross ("+") was shown at the center of the screen for $500 \mathrm{~ms}$.

Stimuli were presented until 10 minutes (practice session) or 30 minutes (main session) passed, such that all stimuli were shown once in random order, generated by shuffling in Presentation ${ }^{\circledR}$ software. Afterwards, every stimulus was shown again twice in another random order. Between the first two occurrences of the same stimulus, at least 20 other stimuli were shown. The orientation of the left and right base figure was randomized. the laptop with their left index finger if the stimulus could be rotated into congruence with the left base figure. If the stimulus could be rotated into congruence with the right base figure, participants should press the right mouse button with their right index finger. Participants were asked to answer as quickly and as precisely as possible. orientation, base orientation), and time since start of the session were recorded.

\section{Demographics}

244 A digital questionnaire was used to collect demographic information. Participants were asked

245 about their previous experience with mental rotation (participants had to indicate if they had

246 or had not participated in other mental rotation experiments before), age (in years),

247 sex/gender (male, female, or other), information about their menstrual cycle, physical and musical activity, and handedness. 
250 Participants completed a practice session of 10 minutes and continued with the main session of 30 minutes after a self paced break. Following the main session, participants answered a digital questionnaire regarding demographic data. Total durations of individual sessions were known to participants before the start of the experiment and were shown again before each session. For the duration of the experiment and the questionnaire, participants were alone in the experimental room.

\section{Statistical analysis}

257

The accuracy and time of response of each trial were used as dependent variables and the angular disparity, time (since start of the session), the side of the correct answer, the rotation axis, and previous experience of participants were used as independent variables.

Outliers were determined by a deviance of more than three standard deviations from the mean reaction time of all stimulus pairs with the same rotation angle and were excluded from all analyses and reaction time was additionally only analyzed on correct responses. Moreover, as the rotation axis is not well-defined for the $0^{\circ}$-condition, all analyses including axes were calculated only on the rotated stimuli. For all effects not containing axes, the analysis was calculated twice, once on the dataset including the non-rotated stimuli and once on the dataset of only the rotated stimuli, possibly including effects of axes as moderating effects. By this procedure, 302 of 17900 trials were deemed as outliers. Of the remaining trials, 2073 were incorrect responses and 1142 trials used no rotation.

Statistical analysis was performed according to Jost, Weishäupl, and Jansen (2019) with linear mixed models using lme4 package (version 1.1-21; Bates, Maechler, Bolker, \& Walker, 2015) in R (version 3.5.1; R Core Team, 2018). Model parameters were estimated by maximum likelihood estimation using bobyqa algorithm wrapped by optimx package (version 
273 2018-7.10; Nash \& Varadhan, 2011) as optimizer. Model fit was calculated by using

274 likelihood ratio tests to compare models with and without the fixed effect of interest. The

275 resulting p-values were compared to a significance level of .05. For multiple comparisons of

276 the same variables, the significance level was Bonferoni corrected. Visual inspection of

277 residual plots did not reveal deviations from homoscedasticity or normality in any model.

278 Where possible, we report both the unstandardized effect sizes and confidence

279 intervals calculated by using parametric bootstrapping with 1000 simulations in line with

280 recommendations of Baguley (2009) and Pek and Flora (2018). While standardized effect

281 sizes are routinely used for power analysis and meta analyses, unfortunately there does not

282 exist an agreed upon way to compute standardized effect sizes in linear mixed models

283 (Feingold, 2009; Hedges, 2007; Rights \& Sterba, 2019). Nevertheless, linear mixed models

284 offer several advantages over traditional use of ANOVAs. For example, linear mixed models

285 allow simultaneous analysis of by-participant and by-item variances and thus eliminating the

286 need to average over participants or items, while also allowing analysis of unbalanced data

287 and achieving higher statistical power (Barr et al., 2013, Hilbert et al., 2019). These allowed

288 the experiment to be controlled by time with participants finishing different numbers of trials

289 and enabled the analysis of time as an independent variable.

290 Model building was based on the research of Barr et al. (2013) and Bates, Kliegl,

291 Vasishth, and Baayen (2015), starting with a model with random intercepts and slopes for

292 every appropriate fixed effect and reducing the model complexity by dropping non-

293 significant variance components. Non-significant fixed effects were further stepwise removed

294 from the model, such that effects which least decreased model fit were removed first and a

295 model containing only significant fixed effects remained. Non-significant effects were then

296 tested for an improvement of model fit by inclusion in the resulting model, while significant

297 effects were tested for worsening of model fit by exclusion of the effect. Main effects for 
significant interactions were tested separately by splitting the interaction. The resulting models for each parameter are described in the results section.

As there is ongoing discussion about model selection based on theory or data or preferring complex instead of simpler models, we expect future research to cast doubt on the optimality of the currently suggested models. Although models without random slopes seem too anti-conservative (Barr et al., 2013) we report results of these simplest models for easier comparison.

\section{Results}

\section{Descriptive statistics}

307 Means and standard deviations for both reaction time and accuracy are provided in table 2 for both side and degree as the main effects in question. Due to the time-controlled nature of the experiment, participants performed different number of trials. Average reaction time and accuracy are calculated for every participant. Mean and standard deviations are then reported

311 between participants. Behavioral data for other conditions and summarized demographic data

312 can be found at https://github.com/LeonardoJost/MRExperiment.

$$
\text { Please insert table } 2 \text { about here }
$$

\section{$314 \quad$ Reaction time}

315 For the analysis of reaction time on the dataset of only rotated stimuli, model building resulted in a model with random intercepts and random slopes for degree and time (since start

317 of the session) by participant and random intercepts by model. The interactions

318 degree*time*side and degree*axis*side as well as the included two-way interactions and

319 main effects, and the effects of previous experience were analyzed as fixed effects.

320 Significant effects were found for experience, degree*time, degree*side, degree*axis, degree, 
and time. Reaction time improved with experience and time and increased with angular disparity. The effect of angular disparity was lower with increased time, for answers on the right side, and for rotations around the z-axis. (See table 3)

\section{Please insert table 3 about here}

In a model without random slopes, the effects of experience $\left(\chi^{2}(1)=0.13, p=.719\right)$ and degree* side $\left(\chi^{2}(1)=3.53, p=.060\right)$ were not significant, but otherwise no differences in significance were found.

For the analysis of reaction time on the dataset of all stimuli, model building resulted in a model with random intercepts and random slopes for degree and time by participant and random intercepts by model. The interaction degree*time*side and the effects of previous experience were analyzed as fixed effects. Significant effects were found for experience,

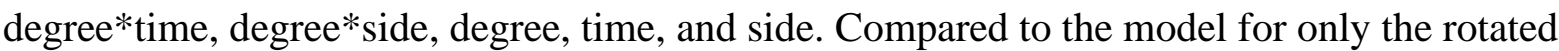
stimuli, reaction times were significantly slower for answers on the right side, but otherwise no differences in significance were found. (See table 4)

\section{Please insert table 4 about here}

In a model without random slopes, the effects of experience $\left(\chi^{2}(1)=0.11, p=.740\right)$ and side $\left(\chi^{2}(1)=3.65, p=.056\right)$ were not significant, but otherwise no differences in significance were found.

\section{Accuracy}

Accuracy was analyzed by a general linear mixed model, which used a binomial distribution. For the analysis on the dataset of only rotated stimuli, model building resulted in a model with random intercepts and random slopes for degree, time (since start of the session), and axis by participant and random intercepts and random slopes for time by model. The interactions degree*time* side and degree*axis*side and the effects of previous experience 
were analyzed as fixed effects. Significant effects were found for degree*axis, degree, and time. Accuracy was significantly higher with increased time and significantly lower with increased angular disparity. The effect of degree was lower for rotations around the z-axis.

\section{$348 \quad($ See table 5)}

\section{Please insert table 5 about here}

In a model without random slopes, the effects of degree*time $\left(\chi^{2}(1)=5.12, p=.024\right)$, degree* side $\left(\chi^{2}(1)=3.94, p=.047\right)$, and axis $\left(\chi^{2}(1)=7.41, p=.006\right)$ were significant, but otherwise no differences in significance were found.

For the analysis of accuracy on the dataset of all stimuli, model building resulted in a model with random intercepts and random slopes for degree and time by participant and random intercepts and random slopes for time by model. The interaction degree*time*side and the effects of previous experience were analyzed as fixed effects. Significant effects were found for degree*side, degree, and time. Compared to the model for only the rotated stimuli the decrease of accuracy with increasing angular disparity was significantly smaller for answers on the right side, but otherwise no differences in significance were found. (See table 6)

In a model without random slopes, the effect of degree*time $\left(\chi^{2}(1)=7.39, p=.007\right)$ was significant and the effect of time $\left(\chi^{2}(1)=3.58, p=.058\right)$ was not significant, but otherwise no differences in significance were found.

As we observed nonlinear behavior in the accuracy data averaged by participant, we performed separate comparisons by degree for both rotation axis and the correct side. For this, we used a model with random intercepts and random slopes for time by both participant and model. As fixed effects, we included both axis and side for all angles for comparison purposes. At $0^{\circ}$ answers on the right side were significantly less accurate than answers on the 
left side, while at $180^{\circ}$ they were significantly more accurate. No differences were found at $45^{\circ}, 90^{\circ}$, and $135^{\circ}$. Rotations around the z-axis produced significantly more accurate answers at $135^{\circ}$ and $180^{\circ}$ compared to rotations around the y-axis. (See table 7)

Please insert table 7 about here

\section{Linearity of data}

As we observed nonlinear behavior in the data, we analyzed the influence of additional polynomial functions of angular disparity on both reaction time and accuracy. In these cases, a significant improvement of model fit was found for quadratic and cubic polynomials. All estimated polynomials were monotonously increasing for reaction time and monotonously decreasing for accuracy in the value range of interest. (See table 8)

\section{Please insert table 8 about here}

\section{Discussion}

Our study provides insight that the newly developed test is applicable for measuring mental rotation ability. With this test all stimuli can be analyzed, although small differences between the sides of the correct base figure persist. We have found some minor differences in reaction time but some larger differences in accuracy. While the behavior of reaction time and accuracy by degree shows changes by side and with different rotation axes, the overall results are comparable to the behavioral data of "same" stimuli in the design of Shepard and Metzler (1971) with two images regarding effects of degree. That is, for both sides of the congruent base figure we observe the behavior expected of "same" stimuli. Moreover, if only one base figure were used to solve the task, we should have found reaction time differences comparable to those between "same" and "different" trials in two-figure mental rotation tasks. As this is not the case, we assume that the unique $0^{\circ}$-position is used by participants and the same mental rotation process is measured in all trials. 
Reaction time increases with larger angles and decreases over time, with larger improvements at larger angles. We have found differences in the interaction of degree and side, but on both sides our results show the expected increase of reaction time at larger angles. Moreover, the differences by side are smaller than by axis, which are typically assumed to be the same.

399 Possibly of the same magnitude are effects of rotation direction, which were not analyzed and are also typically grouped together. Additionally, the improvement over time at all angles did not differ by side, which leads us to the assumption, that the same mental transformation processes are used on both sides. This is also supported by the lower reaction times of participants with previous experience with the two-figure layout, although learning effects are not fully understood (Heil, Rösler, Link, \& Bajric, 1998; Meneghetti et al., 2017; Rahe, Ruthsatz, Jansen, \& Quaiser-Pohl, 2019; Uttal et al., 2013). as well as differences by axis. Compared to reaction time, accuracy shows no significant differences for previous experience and for the interaction of degree and time, but results 409 point in the same direction as for reaction time. In the case of side and axis, the results indicate higher accuracies at lower reaction times and cannot be justified by speed-accuracy

411 tradeoffs. The differences in accuracy at $0^{\circ}$ are quite surprising, as no mental rotation is 412 necessary, yet average accuracy was only at $91 \%$ for answers on the right side (compared to $41397 \%$ for the left side). One possible explanation might be the left to right reading direction 414 and thus figures were possibly also compared in that order, although reaction time differences 415 would be better explained by this than accuracy differences. Also, we have observed a quite 416 unexpected increase in accuracy for rotations around the z-axis at larger angles. While this 417 might be influenced by the layout of the figures or the base angles of the figures, an 418 interaction with the side of answer was not significant. A possible influence can be seen in 
419 figure 4 for one model, but the other models show a similar position of the characteristic arm.

420 For a rotation around the z-axis, the characteristic arm of the figure stays close to the two

421 base figures, whereas for a rotation around the $y$-axis the optical distance between stimulus

422 and base figures increases. By the same matter, there is a possible overlap of the figure with

423 the fixation cross between trials for rotations around the z-axis. Another difference can be

424 seen in the base angles, which produce different directions of the front- and top-facing arms,

425 which would point straight forward or upwards if no base angles were used. As these

426 explanations are speculative, it remains unclear, how and why accuracy differences by axis

427 occur. Further research needs to be done on reproducibility and explanations of these

428 phenomena and accuracy should be interpreted with more caution.

429 Similarly as for reaction time, overall accuracy patterns show smaller differences by

430 side than by axis and no interactions with time, which can be interpreted in support of the

431 notion that the same mental transformations are performed on both sides as in the two-figure

432 case.

Average reaction times are also similar to other studies using comparable stimuli but

434 different designs. For example, Jost, Weishäupl, and Jansen (2019) used two main sessions of

43530 minutes duration on different days and found on average slightly lower reaction times.

436 Meneghetti et al. (2017) used multiple training sessions of approximately 35 minutes duration

437 and found similar reaction time in their second session. Thus, our layout enables the

438 completion of more analyzable tasks in time and achieves the desired increase in power.

\section{Nonlinearity}

440 Although residual plots for models using linear dependency of reaction time on degree show

441 no visual deviations, significant higher order polynomial relationships were observed.

442 Whereas the original paper of Shepard and Metzler (1971) showed perfect linearity in 
443 reaction time, this is not unanimously reproduced, e.g. in Jolicœur, Regehr, Smith, and Smith

444 (1985), and, although often not discussed, inspection of the data in other work rarely shows

445 perfect linearity. Thus, we believe that nonlinearity is not a major concern in discussing if our

446 setup can be used to analyze the same mental processes as the original design. While the

447 linearity allows a simple theoretical framework, namely a fixed mental rotation speed, we

448 have no simple explanation for the nonlinearity. Differing reaction time-accuracy tradeoffs

449 might be one reason, but different strategies analyzing mirrorings instead of rotation might

450 also contribute. In our opinion, the important findings are that reaction time increases and

451 accuracy decreases with increasing angular disparity and deviations from linearity do not

452 show large effects. Until further research is available, we believe, if nonlinearity can be

453 observed in the data, further investigating interactions should be warranted even if they are

454 not significant, e.g. in our data in the case of accuracy differences by side for angular

455 disparity of $180^{\circ}$.

456 Limitations

457 One limitation is the inference of mental rotation only from behavioral data. Although it 458 seems plausible, we cannot know if strategies incorporate the usage of both stimuli or if 459 stimuli are actually rotated in the mind from this behavioral data alone and thus cannot infer

460 that the same strategies are used in our design as in the two-figure case. However, the 461 investigation of strategies was not the main goal of our study. Moreover, effects of increasing 462 mental fatigue in time as well as effects of the shape and layout of the cube figures could 463 have influenced our results.

\section{Conclusion and outlook}

465 In conclusion, we have developed a new layout for chronometric mental rotation tests

466 utilizing three figures. While small differences in performance need to be further analyzed, 
overall our design is appropriate for analyzing mental rotation performance. The new layout offers an increase of power while also solving possible problems with analysis of accuracy.

Our theoretical framework of layout parameters as well as the described stimulus library provide possibilities for future research and implementation.

472 first to analyze the progress of participants during the experiment. While we analyzed the 473 absolute improvement in ms and the proportion of correct answers with more time spent on the task, future research could also analyze improvements relative to participants mean performance or with the number of items solved. We also found performance improvements for participants who had experience with mental rotation experiments. There is potential for future work to specify the variable experience more precisely and further explore the relationship to performance.

Further studies could also explore influences on mental rotation performance of different cube figures or different versions of the same figures. This includes the expansion in the three dimensions, the alignment with the origin, and coloring parameters. Design parameters could also be varied in attempt to force different strategies and eye tracking or EEG could be used to analyze the actual use of strategies. (1978) type by adding a mirrored or structurally different base figure to the right of the stimuli, but as in the case of our work, different setups are possible. (Although single item performance in Vandenberg and Kuse tests cannot be tracked and the need is possibly lessened.) Moreover, other pattern recognition tasks could also apply similarly inspired designs. 


\section{Declarations}

491 Ethical standards. The experiment was conducted according to ethical declaration of Helsinki.

492 Ethical approval for this study was not required in accordance with the conditions outlined by the

493 German Research Society (DFG) where research that carries no additional risk beyond daily activities

494 does not require Research Ethics Board Approval. We communicated all considerations necessary to

495 assess the question of ethical legitimacy of the study.

496 Informed Consent. Informed consent was obtained from all individual participants included in the 497 study.

498 Acknowledgements. We thank Arne Engelhardt (University of Regensburg) for his support with data 499 collection. We thank Philipp Hofmann and Markus Siebertz (University of Regensburg) for their 500 support with testing the stimulus library.

501 Data availability. The data that support the findings of this study are available at https://osf.io/dr9mv/

502 (DOI 10.17605/OSF.IO/DR9MV). The experiment was not preregistered.

503 Competing interests. The authors declare no competing interests.

504 Funding. This research did not receive any specific grant from funding agencies in the public, 505 commercial, or not-for-profit sectors.

506 Author contributions. All authors contributed to the study conception and design. Material 507 preparation, data collection, and data analysis was performed by Leonardo Jost. The manuscript was 508 written by Leonardo Jost and Petra Jansen. Both authors read and approved the final manuscript.

\section{References}

511 Baguley, T. (2009). Standardized or simple effect size: What should be reported?. British $512 \quad$ journal of psychology, 100(3), 603-617.

513 Barr, D. J., Levy, R., Scheepers, C., \& Tily, H. J. (2013). Random effects structure for confirmatory hypothesis testing: Keep it maximal. Journal of memory and language, 68(3), 255-278.

Bates, D., Maechler, M., Bolker, B., \& Walker, S. (2015). Fitting Linear Mixed-Effects Models Using lme4. Journal of Statistical Software, 67(1), 1-48. doi:10.18637/jss.v067.i01.

Bates, D., Kliegl, R., Vasishth, S., \& Baayen, H. (2015). Parsimonious mixed models. arXiv preprint arXiv:1506.04967. 
Boone, A. P., \& Hegarty, M. (2017). Sex differences in mental rotation tasks: Not just in the mental rotation process!. Journal of Experimental Psychology: Learning, Memory, and Cognition, 43(7), 1005.

Brysbaert, M., \& Stevens, M. (2018). Power analysis and effect size in mixed effects models: A tutorial. Journal of Cognition, 1(1).

Faul, F., Erdfelder, E., Lang, A. G., \& Buchner, A. (2007). G* Power 3: A flexible statistical power analysis program for the social, behavioral, and biomedical sciences. Behavior research methods, 39(2), 175-191.

Feingold, A. (2009). Effect sizes for growth-modeling analysis for controlled clinical trials in the same metric as for classical analysis. Psychological methods, 14(1), 43.

Geary, D. C., Saults, S. J., Liu, F., \& Hoard, M. K. (2000). Sex Differences in Spatial Cognition, Computational Fluency, and Arithmetical Reasoning. Journal of Experimental Child Psychology, 77(4), 337-353. https://doi.org/10.1006/jecp.2000.2594.

Hausmann, M. (2014). Arts versus science - Academic background implicitly activates gender stereotypes on cognitive abilities with threat raising men's (but lowering women's) performance. Intelligence, 46, 235-245. http://dx.doi.org/10.1016/j.intell.2014.07.004.

Hedges, L. V. (2007). Effect Sizes in Cluster-Randomized Designs. Journal of Educational and Behavioral Statistics, 32(4), 341-370. https://doi.org/10.3102/1076998606298043

Heil, M., Rösler, F., Link, M., \& Bajric, J. (1998). What is improved if a mental rotation task is repeated-the efficiency of memory access, or the speed of a transformation routine?. Psychological Research, 61(2), 99-106.

Hilbert, S., Stadler, M., Lindl, A., Naumann, F., \& Bühner, M. (2019). Analyzing longitudinal intervention studies with linear mixed models. TPM - Testing, Psychometrics, Methodology in Applied Psychology, 26(1), 101-119. https://doi.org/10.4473/TPM26.1.6

Jansen-Osmann, P., \& Heil, M. (2007). Suitable stimuli to obtain (no) gender differences in the speed of cognitive processes involved in mental rotation. Brain and Cognition, 64(3), 217-227.

Jolicœur, P., Regehr, S., Smith, L. B., \& Smith, G. N. (1985). Mental rotation of representations of two-dimensional and three-dimensional objects. Canadian Journal of Psychology/Revue canadienne de psychologie, 39(1), 100. 
555

556

557

558

559

560

561

562

563

564

565

566

567

568

569

570

571

572

573

574

575

576

577

578

579

580

581

582

583

584

585

586

587

Jost, L., Weishäupl, A., \& Jansen, P. (2019). Interactions between simultaneous aerobic exercise and mental rotation. Manuscript submitted for publication.

Khooshabeh, P., \& Hegarty, M. (2010, March). Representations of shape during mental rotation. In AAAI Spring Symposium: Cognitive Shape Processing.

Kozhevnikov, M., \& Hegarty, M. (2001). A dissociation between object manipulation spatial ability and spatial orientation ability. Memory \& Cognition, 29(5), 745-756.

Krüger, M., \& Krist, H. (2009). Imagery and motor processes-When are they connected? The mental rotation of body parts in development. Journal of Cognition and Development, 10(4), 239-261.

Meneghetti, C., Cardillo, R., Mammarella, I. C., Caviola, S., \& Borella, E. (2017). The role of practice and strategy in mental rotation training: transfer and maintenance effects. Psychological research, 81(2), 415-431.

Moè, A. (2016). Does experience with spatial school subjects favour girls' mental rotation performance? Learning and Individual Differences, 47, 11-16. http://dx.doi.org /10.1016/j.lindif.2015.12.007

Moè, A., Jansen, P., \& Pietsch, S. (2018). Childhood preference for spatial toys. Gender differences and relationships with mental rotation in STEM and non-STEM students. Learning and Individual Differences, 68, 108-115.

Nash, J. C., \& Varadhan, R. (2011). Unifying optimization algorithms to aid software system users: optimx for R. Journal of Statistical Software, 43(9), 1-14.

Pek, J., \& Flora, D. B. (2018). Reporting effect sizes in original psychological research: A discussion and tutorial. Psychological Methods, 23(2), 208.

Peters, M., \& Battista, C. (2008). Applications of mental rotation figures of the Shepard and Metzler type and description of a mental rotation stimulus library. Brain and cognition, 66(3), 260-264.

Rahe, M., Ruthsatz, V., Jansen, P., \& Quaiser-Pohl, C. (2019). Different practice effects for males and females by psychometric and chronometric mental-rotation tests. Journal of Cognitive Psychology, 31(1), 92-103.

Rights, J. D., \& Sterba, S. K. (2019). Quantifying explained variance in multilevel models: An integrative framework for defining R-squared measures. Psychological methods, 24(3), 309.

R Core Team (2018). R: A language and environment for statistical computing. R Foundation for Statistical Computing, Vienna, Austria. URL https://www.R-project.org/. 
614

Shepard, R. N., \& Metzler, J. (1971). Mental rotation of three-dimensional objects. Science, 171(3972), 701-703.

Shepard, S., \& Metzler, D. (1988). Mental rotation: effects of dimensionality of objects and type of task. Journal of experimental psychology: Human perception and performance, $14(1), 3$.

Stanislaw, H., \& Todorov, N. (1999). Calculation of signal detection theory measures. Behavior research methods, instruments, \& computers, 31(1), 137-149.

Uttal, D. H., Meadow, N. G., Tipton, E., Hand, L. L., Alden, A. R., Warren, C., \& Newcombe, N. S. (2013). The malleability of spatial skills: A meta-analysis of training studies. Psychological bulletin, 139(2), 352.

Vandenberg, S. G., \& Kuse, A. R. (1978). Mental rotations, a group test of three-dimensional spatial visualization. Perceptual and motor skills, 47(2), 599-604.

Voyer, D., \& Jansen, P. (2016). Sex differences in chronometric mental rotation with human bodies. Psychological research, 80(6), 974-984.

Voyer, D., Jansen, P., \& Kaltner, S. (2017). Mental rotation with egocentric and object-based transformations. The Quarterly Journal of Experimental Psychology, 70(11), 23192330.

Wohlschläger, A. (2001). Mental object rotation and the planning of hand movements. Perception \& Psychophysics, 63(4), 709-718.

Wohlschläger, A., \& Wohlschläger, A. (1998). Mental and manual rotation. Journal of Experimental Psychology: Human perception and performance, 24(2), 397.

Xie, F., Zhang, L., Chen, X., \& Xin, Z. (2019). Is Spatial Ability Related to Mathematical Ability: a Meta-analysis. Educational Psychology Review. https://doi.org/10.1007/s10648-019-09496-y

Zacks, J. M., \& Tversky, B. (2005). Multiple systems for spatial imagery: Transformations of objects and bodies. Spatial Cognition and Computation, 5(4), 271-306. 


\section{Tables}

617 Table 1. Parameters for generation of cube figures and values used in the experiment.

\begin{tabular}{lll}
\hline Parameter group & Parameter & value \\
\hline Color options & Background color & black \\
& Border color & black \\
& Face color & white, grey \\
Sizing and formatting & Cube Diameter & $70 \mathrm{px}$ \\
& Image size & $720 \mathrm{px} * 720 \mathrm{px}$ \\
& File format & png \\
& Centering & none \\
Model properties & Base orientations & a,b \\
& Models & Peters and Battista $(2008), 1-16$ \\
& Base rotation angles $(\mathrm{x}, \mathrm{y}, \mathrm{z})$ & $-30^{\circ}, 0^{\circ}, 15^{\circ}$ \\
& Angle difference & $45^{\circ}$ \\
& Rotation axes & $\mathrm{y}, \mathrm{z}$ \\
\hline
\end{tabular}

618

619 Table 2. Mean (SD) of behavioral data, separated by the side of the congruent base figure

620 (columns) and degree (rows). Reaction time is reported in ms, accuracy in the proportion of

621 correct answers. Mean reaction time is calculated for all correctly answered trials of each

622 participant and then averaged over all participants. Mean accuracy is calculated for all trials

623 of each participant and then averaged over all participants

\begin{tabular}{lllll}
\hline & Reaction time & \multicolumn{3}{l}{ Accuracy } \\
\hline & Left & Right & Left & Right \\
$0^{\circ}$ & $1824(643)$ & $2074(578)$ & $.97(.04)$ & $.91(.12)$ \\
$45^{\circ}$ & $2830(942)$ & $2896(938)$ & $.92(.09)$ & $.92(.09)$ \\
$90^{\circ}$ & $3664(1047)$ & $3765(1151)$ & $.87(.11)$ & $.87(.12)$ \\
$135^{\circ}$ & $4252(1477)$ & $4343(1467)$ & $.85(.13)$ & $.86(.10)$ \\
$180^{\circ}$ & $4804(1467)$ & $4704(1525)$ & $.81(.15)$ & $.86(.11)$ \\
\hline
\end{tabular}

624 
626 Table 3. Statistical analysis of reaction time including axis on the dataset of only rotated

627 stimuli. The values for degree and time (since start of the session) represent estimated

628 changes corresponding to changes of $100^{\circ}$ and 30 minutes of testing time. The values for

629 side=right, axis $=\mathrm{z}$, and experience $=$ no are all relative to the other respective condition. The

630 intercept in this model represents the estimated reaction time in $\mathrm{ms}$ at time $=0$, degree $=0$,

631 side=left, axis=y, experience=yes.

\begin{tabular}{llllll}
\hline Variable & Estimate & SE & Test statistic & $\mathrm{p}$ & 95\% CI \\
\hline Intercept & 2098.64 & 201.31 & & & $1686.14,2487.78$ \\
Degree*Time & -671.04 & 98.53 & $\chi^{2}(1)=46.28$ & $<.001$ & $-863.22,-474.91$ \\
Degree*(Side=right) & -113.56 & 57.00 & $\chi^{2}(1)=3.97$ & .046 & $-223.75,-10.08$ \\
Degree*(Axis=Z) & -374.20 & 56.88 & $\chi^{2}(1)=43.21$ & $<.001$ & $-481.64,-272.71$ \\
Degree*(Time=0)* & 2062.09 & 114.75 & & & $1828.19,2298.92$ \\
(Side=left)*(Axis=Y) & & & & & \\
(Degree=0)*Time & -492.00 & 159.02 & & & $-782.20,-188.61$ \\
(Degree=0)*(Side=right) & 149.81 & 63.55 & & & $32.34,269.56$ \\
(Degree=0)*(Axis=Z) & 387.88 & 63.46 & & & \\
Experience=no & 476.49 & 203.07 & $\chi^{2}(1)=4.98$ & .026 & $55.34,862.66$ \\
Main Effects: & & & & & \\
Time & -1177.24 & 123.27 & $\chi^{2}(1)=47.95$ & $<.001$ & $-1417.51,-908.96$ \\
Side=right & 34.65 & 26.41 & $\chi^{2}(1)=1.72$ & .190 & $-21.39,90.13$ \\
Axis=Z & 8.14 & 26.41 & $\chi^{2}(1)=0.10$ & .758 & $-41.39,61.51$ \\
Degree & 1457.13 & 93.63 & $\chi^{2}(1)=78.83$ & $<.001$ & $1272.79,1648.52$ \\
Nonsignificant Effects: & & & & & \\
Degree*Side*Axis & & & $\chi^{2}(1)=0.11$ & .746 & \\
Degree*Side*Time & & & $\chi^{2}(1)=0.74$ & .389 & \\
Side*Axis & & & $\chi^{2}(1)=2.55$ & .111 & \\
Time*Side & & & $\chi^{2}(1)=0.24$ & .626 & \\
\hline
\end{tabular}

632 
634 Table 4. Statistical analysis of reaction time excluding axis on the dataset of all stimuli. The

635 values for degree and time (since start of the session) represent estimated changes

636 corresponding to changes of $100^{\circ}$ and 30 minutes of testing time. The values for side=right

637 and experience $=$ no are all relative to the other respective condition. The intercept in this

638 model represents the estimated reaction time in $\mathrm{ms}$ at time $=0$, degree $=0$, side $=\mathrm{left}$,

639 experience=yes.

\begin{tabular}{llllll}
\hline Variable & Estimate & SE & Test statistic & $\mathrm{p}$ & $95 \%$ CI \\
\hline Intercept & 2207.46 & 172.91 & & & $1870.43,2539.50$ \\
Degree*Time & -686.97 & 83.58 & $\chi^{2}(1)=67.33$ & $<.001$ & $-862.07,-523.55$ \\
Degree*(Side=right) & -149.63 & 48.20 & $\chi^{2}(1)=9.63$ & .002 & $-244.49,-53.33$ \\
$\begin{array}{l}\text { Degree*(Time=0)* } \\
\text { (Side=left) }\end{array}$ & 1994.49 & 107.73 & & & $1787.55,2192.85$ \\
(Degree=0)*Time & -473.26 & 141.90 & & & $-737.29,-195.83$ \\
(Degree=0)*(Side=right) & 191.26 & 51.85 & & & $91.52,291.70$ \\
Experience=no & 323.01 & 158.03 & $\chi^{2}(1)=3.89$ & .049 & $1.76,651.41$ \\
Main Effects: & & & & & \\
Time & -1125.96 & 117.44 & $\chi^{2}(1)=48.18$ & $<.001$ & $-1354.56,-881.59$ \\
Side=right & 50.00 & 24.87 & $\chi^{2}(1)=4.04$ & .044 & $6.13,101.95$ \\
Degree & 1555.60 & 94.60 & $\chi^{2}(1)=82.80$ & $<.001$ & $1359.07,1755.70$ \\
Nonsignificant Effects: & & & & & \\
Degree*Side*Time & & & $\chi^{2}(1)=0.32$ & .570 & \\
Time*Side & & & $\chi^{2}(1)=0.41$ & .521 & \\
\hline
\end{tabular}

640

641 
642 Table 5. Statistical analysis of accuracy including axis on the dataset of only rotated stimuli

643 using a binomial distribution. The values for degree and time (since start of the session)

644 represent estimated changes corresponding to changes of $100^{\circ}$ and 30 minutes of testing time.

645 The values for side $=$ right, axis $=\mathrm{z}$, and experience $=$ no are all relative to the other respective

646 condition. The intercept in this model represents the estimate at time $=0$, degree $=0$, axis $=y$.

\begin{tabular}{llllll}
\hline Variable & Estimate & SE & Test statistic & $\mathrm{p}$ & $95 \%$ CI \\
\hline Intercept & 3.14 & 0.25 & & & $2.67,3.65$ \\
Degree*(Axis=Z) & 0.63 & 0.11 & $\chi^{2}(1)=31.38$ & $<.001$ & $0.41,0.86$ \\
Degree*(Axis=Y) & -1.05 & 0.11 & & & $-1.28,-0.84$ \\
(Degree=0)*(Axis=Z) & -0.60 & 0.15 & & & $-0.92,-0.32$ \\
Time & 0.34 & 0.16 & $\chi^{2}(1)=4.52$ & .033 & $0.02,0.66$ \\
Main Effects: & & & & & \\
Axis=Z & 0.12 & 0.08 & $\chi^{2}(1)=2.06$ & .151 & $-0.04,0.29$ \\
Degree & -0.75 & 0.09 & $\chi^{2}(1)=45.61$ & $<.001$ & $-0.93,-0.58$ \\
Nonsignificant Effects: & & & & & \\
Side=right & 0.10 & 0.05 & $\chi^{2}(1)=3.55$ & .059 & $-0.00,0.20$ \\
Degre**Side*Axis & & & $\chi^{2}(1)=0.57$ & .450 & \\
Side*Axis & & & $\chi^{2}(1)=0.09$ & .761 & \\
Degree*Side*Time & & & $\chi^{2}(1)=0.00$ & .955 & \\
Degre**Time & & & $\chi^{2}(1)=0.93$ & .335 & \\
Degree*Side & & & $\chi^{2}(1)=3.83$ & .050 & \\
Time*Side & & & $\chi^{2}(1)=0.88$ & .348 & \\
Experience=no & -0.48 & 0.29 & $\chi^{2}(1)=2.71$ & .100 & $-1.03,0.07$ \\
\hline
\end{tabular}

647 
648 Table 6. Statistical analysis of accuracy excluding axis on the dataset of all stimuli using a

649 binomial distribution. The values for degree and time (since start of the session) represent

650 estimated changes corresponding to changes of $100^{\circ}$ and 30 minutes of testing time. The

651 values for side=right and experience=no are all relative to the other respective condition. The

652 intercept in this model represents the estimate at time $=0$, degree $=0$, side $=$ left.

\begin{tabular}{llllll}
\hline Variable & Estimate & SE & Test statistic & $\mathrm{p}$ & $95 \%$ CI \\
\hline Intercept & 2.99 & 0.21 & & & $2.60,3.46$ \\
Degree*(Side=right) & 0.39 & 0.10 & $\chi^{2}(1)=15.76$ & $<.001$ & $0.21,0.61$ \\
Degree*(Side=left) & -0.93 & 0.09 & & & $-1.12,-0.74$ \\
(Degree=0)*(Side=right) & -0.37 & 0.11 & & & $-0.62,-0.16$ \\
Time & 0.36 & 0.16 & $\chi^{2}(1)=4.74$ & .030 & $0.03,0.70$ \\
Main Effects: & & & & & \\
Side=right & 0.05 & 0.05 & $\chi^{2}(1)=1.19$ & .276 & $-0.04,0.15$ \\
Degree & -0.74 & 0.07 & $\chi^{2}(1)=54.40$ & $<.001$ & $-0.89,-0.59$ \\
Nonsignificant Effects: & & & & & \\
Degree*Side*Time & & & $\chi^{2}(1)=0.02$ & .879 & \\
Degree*Time & & & $\chi^{2}(1)=2.77$ & .096 & \\
Time*Side & & & $\chi^{2}(1)=0.78$ & .378 & \\
Experience=no & -0.40 & 0.27 & $\chi^{2}(1)=2.04$ & .154 & $-0.92,0.18$ \\
\hline
\end{tabular}

653

654 
655 Table 7. Statistical analysis of accuracy separated by angle. Intercepts in this model represent 656 the estimate for axis=y, side=left.

\begin{tabular}{|c|c|c|c|c|c|}
\hline Variable & Estimate & $\overline{S E}$ & Test statistic & $\mathrm{p}$ & $95 \% \mathrm{CI}$ \\
\hline \multicolumn{6}{|l|}{$0^{\circ}$} \\
\hline Intercept & 4.31 & 0.38 & & & $3.73,5.35$ \\
\hline Side $=$ right & -1.40 & 0.31 & $\chi^{2}(1)=22.67$ & $<.001$ & $-2.18,-0.79$ \\
\hline \multicolumn{6}{|l|}{$45^{\circ}$} \\
\hline Intercept & 3.06 & 0.22 & & & $2.65,3.48$ \\
\hline Side $=$ right & 0.01 & 0.11 & $\chi^{2}(1)=0.01$ & .933 & $-0.21,0.24$ \\
\hline Axis $=Z$ & -0.15 & 0.11 & $\chi^{2}(1)=1.72$ & .190 & $-0.39,0.08$ \\
\hline \multicolumn{6}{|l|}{$90^{\circ}$} \\
\hline Intercept & 2.33 & 0.21 & & & $1.95,2.76$ \\
\hline Side $=$ right & 0.04 & 0.09 & $\chi^{2}(1)=0.15$ & .701 & $-0.14,0.21$ \\
\hline Axis $=Z$ & -0.19 & 0.09 & $\chi^{2}(1)=3.98$ & .046 & $-0.38,-0.00$ \\
\hline \multicolumn{6}{|l|}{$135^{\circ}$} \\
\hline Intercept & 1.90 & 0.19 & & & $1.53,2.26$ \\
\hline Side $=$ right & 0.03 & 0.09 & $\chi^{2}(1)=0.11$ & .739 & $-0.14,0.21$ \\
\hline Axis $=Z$ & 0.35 & 0.09 & $\chi^{2}(1)=15.59$ & $<.001$ & $0.17,0.53$ \\
\hline \multicolumn{6}{|l|}{$180^{\circ}$} \\
\hline Intercept & 1.42 & 0.20 & & & $1.06,1.85$ \\
\hline Side $=$ right & 0.43 & 0.12 & $\chi^{2}(1)=12.48$ & $<.001$ & $0.21,0.65$ \\
\hline Axis $=Z$ & 0.63 & 0.12 & $\chi^{2}(1)=27.01$ & $<.001$ & $0.40,0.88$ \\
\hline
\end{tabular}

657

658 
659 Table 8. Estimated coefficients of higher order polynomials for the relationship of reaction

660 time and accuracy with angular disparity at units of $100^{\circ}$. Degree describes the degree of the

661 fitted polynomial and $x^{1}-x^{4}$ describe the coefficients. Test statistics were computed in

662 comparison to the polynomial of one lower degree.

\begin{tabular}{llllllll}
\hline Dataset & Degree & $\mathrm{x}^{1}$ & $\mathrm{x}^{2}$ & $\mathrm{x}^{3}$ & $\mathrm{x}^{4}$ & Test statistic & $\mathrm{p}$ \\
\hline Reaction time & 1 & 2062.09 & & & & & \\
including axis & 2 & 2922.18 & -407.6 & & & $\chi^{2}(1)=35.85$ & $<.001$ \\
& 3 & 4948.50 & -2447.06 & 611.08 & & $\chi^{2}(1)=8.66$ & .003 \\
Reaction time & 1 & 1994.49 & & & & \\
excluding axis & 2 & 2689.69 & -371.33 & & & $\chi^{2}(1)=68.09$ & $<.001$ \\
& 3 & 2757.85 & -464.58 & 33.41 & & $\chi^{2}(1)=0.17$ & .682 \\
& 4 & 1863.39 & 2111.76 & -2272.16 & 637.82 & $\chi^{2}(1)=9.28$ & .002 \\
Accuracy & 1 & -1.05 & & & & & \\
including axis & 2 & -2.57 & 0.66 & & & $\chi^{2}(1)=25.29$ & $<.001$ \\
& 3 & -5.60 & 3.65 & -0.88 & & & \\
Accuracy & 1 & -0.93 & & & & $\chi^{2}(1)=5.19$ & .023 \\
excluding axis & 2 & -1.93 & 0.47 & & & $\chi^{2}(1)=0.10$ & .755 \\
& 3 & -1.80 & 0.30 & 0.06 & & & \\
& 4 & 0.42 & -5.02 & 4.47 & -1.17 & $\chi^{2}(1)=3.80$ & .051 \\
\hline
\end{tabular}

663

664 


\section{Figures}

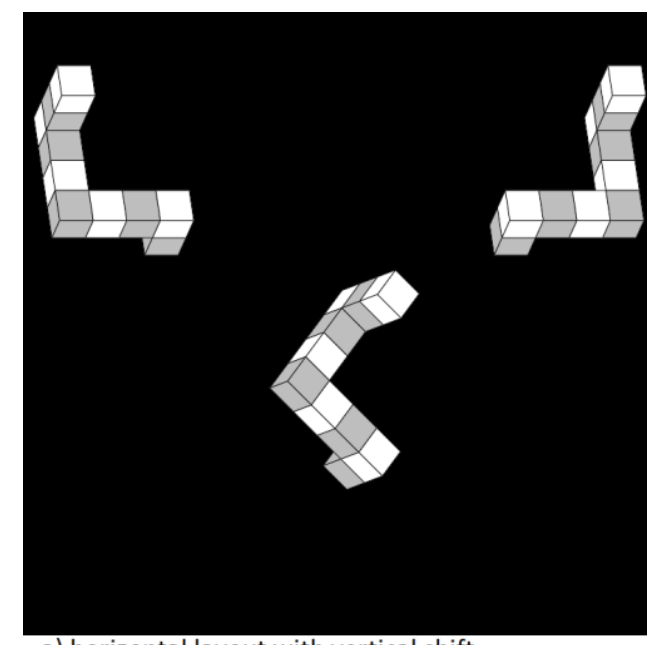

a) horizontal layout with vertical shift

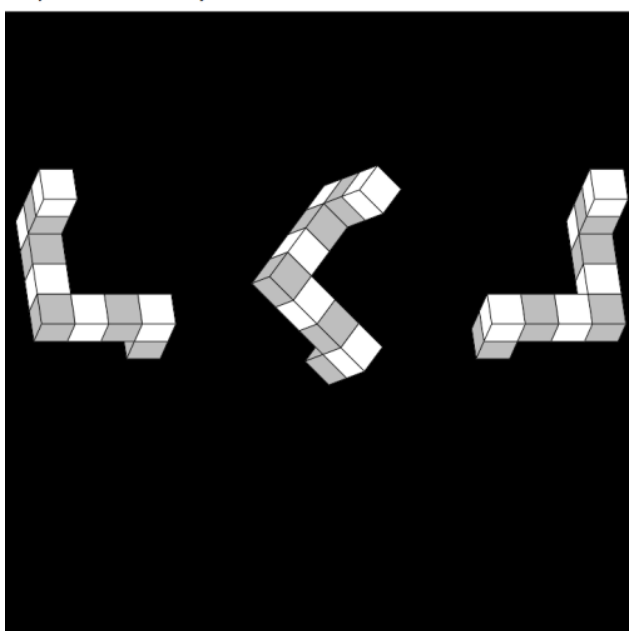

b) horizontal layout

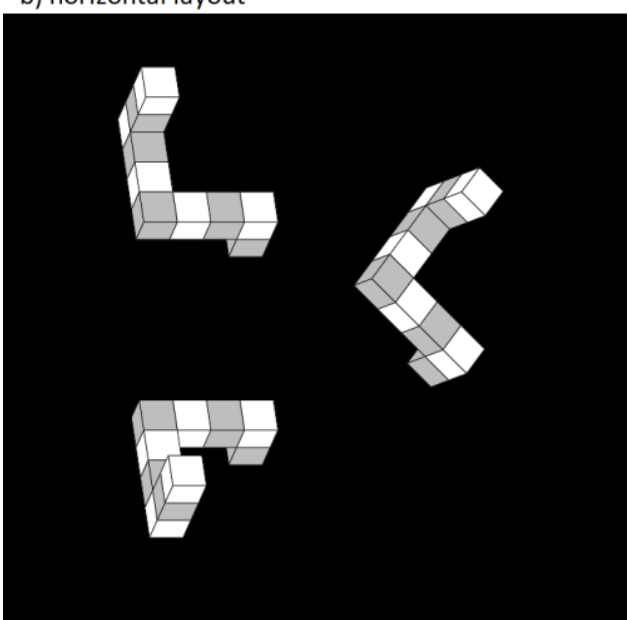

666

c) vertical layout with horizontal shift

667 Figure 1. Depictions of different possible layouts of two base figures and one stimulus figure.

668 The stimulus figure is always rotated by $45^{\circ}$ around the $y$-axis for demonstration while the 669 base figures are mirrored to each other. 


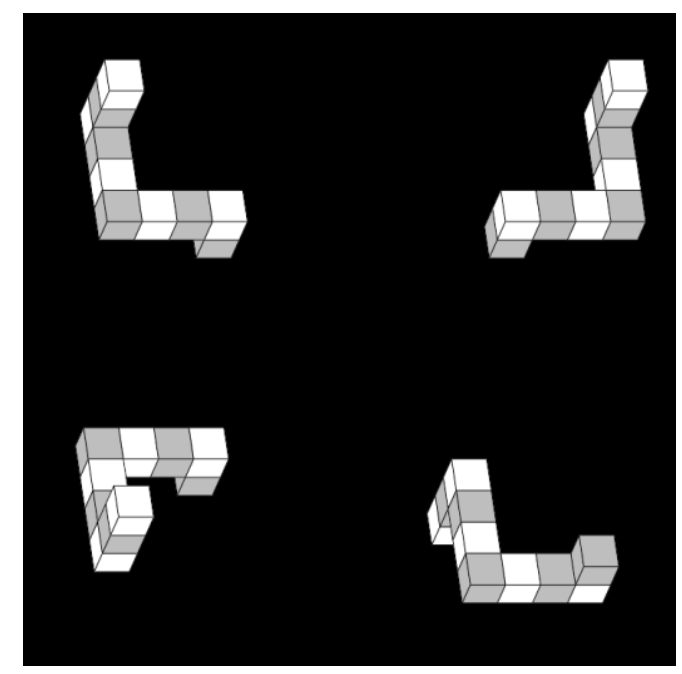

672 Figure 2. Depictions of mirror images across the natural mirroring planes. Top left: base 673 model. Top right: Mirroring across the yz-plane. Bottom left: Mirroring across the xy-plane. 674 Bottom right: Mirroring across the xz-plane.

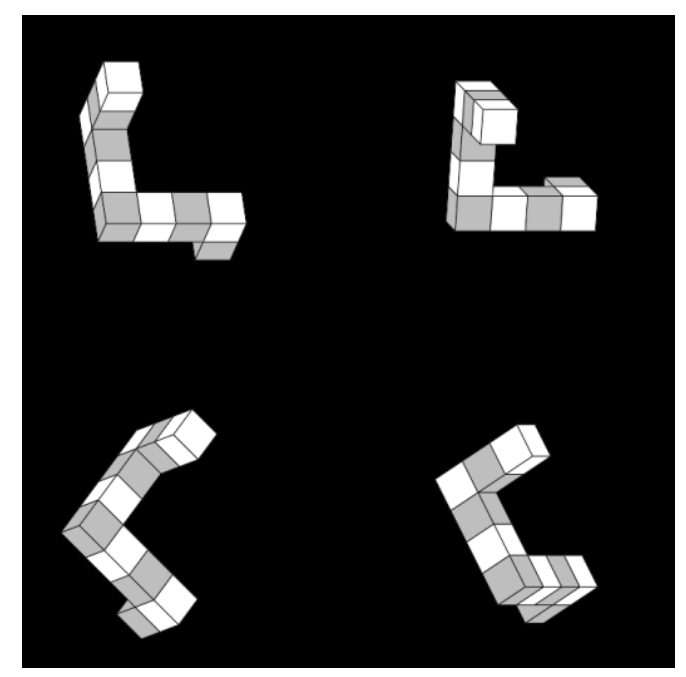

676 Figure 3. Depictions of rotation by $45^{\circ}$ around the natural rotation axes. Top left: Base 677 model. Top right: Rotation around the x-axis. Bottom left: Rotation around the y-axis. 678 Bottom right: Rotation around the z-axis. 

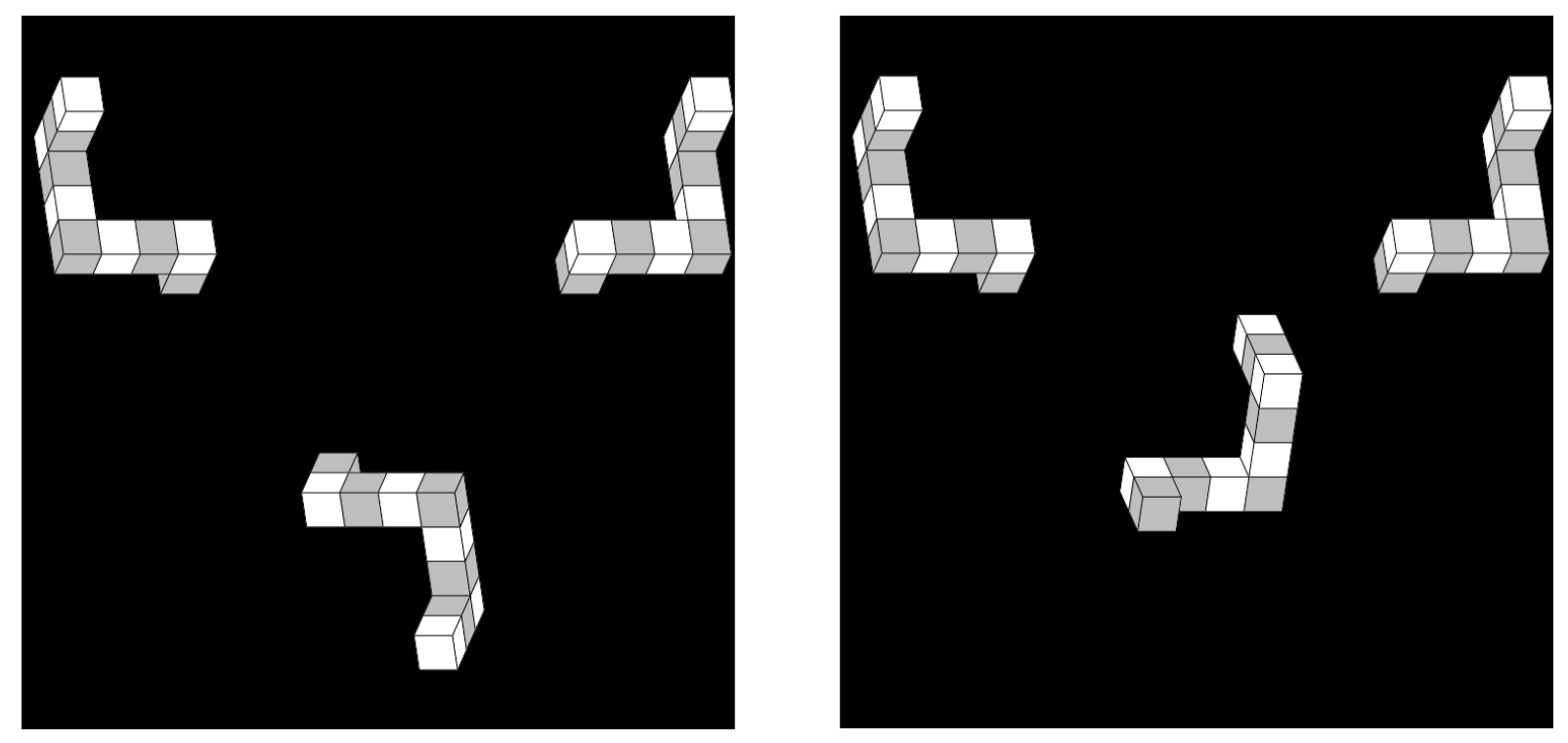

680 Figure 4. Depictions of stimulus figure, that can be rotated into the left base figure by a

681 rotation of $180^{\circ}$ around the y-axis (left) and z-axis(right). 